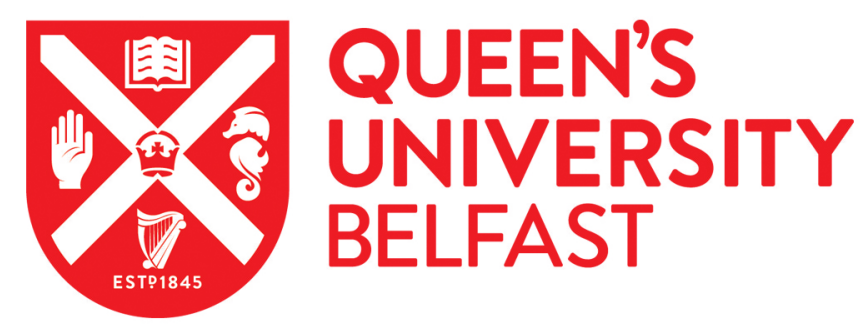

\title{
Experimental measurements of the collisional absorption of XUV radiation in warm dense aluminium
}

Kettle, B., Dzelzainis, T., White, S., Li, L., Dromey, B., Zepf, M., Lewis, C. L. S., Williams, G., Künzel, S.,

Fajardo, M., Dacasa, H., Zeitoun, P., Rigby, A., Gregori, G., Spindloe, C., Heathcote, R., \& Riley, D. (2016).

Experimental measurements of the collisional absorption of XUV radiation in warm dense aluminium. Physical Review E, 94(2), [023203]. https://doi.org/10.1103/PhysRevE.94.023203

Published in:

Physical Review E

Document Version:

Peer reviewed version

Queen's University Belfast - Research Portal:

Link to publication record in Queen's University Belfast Research Portal

Publisher rights

@2016 American Physical Society

\section{General rights}

Copyright for the publications made accessible via the Queen's University Belfast Research Portal is retained by the author(s) and / or other copyright owners and it is a condition of accessing these publications that users recognise and abide by the legal requirements associated with these rights.

Take down policy

The Research Portal is Queen's institutional repository that provides access to Queen's research output. Every effort has been made to ensure that content in the Research Portal does not infringe any person's rights, or applicable UK laws. If you discover content in the Research Portal that you believe breaches copyright or violates any law, please contact openaccess@qub.ac.uk. 


\title{
Experimental measurements of the collisional absorption of XUV radiation in warm dense aluminium
}

\author{
B. Kettle,${ }^{1, *}$ T. Dzelzainis,${ }^{1}$ S. White,${ }^{1}$ L. Li,${ }^{1}$ B. Dromey,${ }^{1}$ M. Zepf,${ }^{1}$ C. L. S. Lewis,${ }^{1}$ G. Williams, ${ }^{2}$ S. Künzel, ${ }^{2}$ M. \\ Fajardo, ${ }^{2}$ H. Dacasa, ${ }^{3}$ Ph. Zeitoun, ${ }^{3}$ A. Rigby,${ }^{4}$ G. Gregori, ${ }^{4}$ C. Spindloe, ${ }^{5}$ R. Heathcote,${ }^{5}$ and D. Riley ${ }^{1}$ \\ ${ }^{1}$ Centre for Plasma Physics, School of Mathematics and Physics, \\ Queen's University Belfast, University Road, Belfast BT7 1NN, UK \\ ${ }^{2}$ GoLP/Instituto de Plasmas e Fusão Nuclear, Instituto Superior Técnico, \\ Universidade de Lisboa, 1049-001 Lisbon, Portugal \\ ${ }^{3}$ Laboratoire d'Optique Appliquée, ENSTA ParisTech, \\ CNRS, Ecole polytechnique, Université Paris-Saclay, \\ 828 bd des Marchaux, 91762 Palaiseau cedex France \\ ${ }^{4}$ Clarendon Laboratory, Parks Road, Oxford, OX1 3PU, UK \\ ${ }^{5}$ Central Laser Facility, Harwell, Oxfordshire, OX11 0QX, UK
}

(Dated: April 21, 2016)

\begin{abstract}
The collisional (or free-free) absorption of soft X-rays in warm dense aluminium remains an unsolved problem. Two competing descriptions of the process exist, one based on a weak scattering model and the other a corrected classical model, both of which show distinctly different behaviours with temperature. Here, we describe novel experimental evidence for the absorption of 25-30 eV photons in solid density warm aluminium $\left(T_{e} \approx 1 \mathrm{eV}\right)$. Radiative x-ray heating from palladium coated $\mathrm{CH}$ foils was used to create the warm dense aluminium samples, and a laser driven high harmonics beam from an argon gas jet provided the probe. The results indicate that of the two, the corrected classical model is better at predicting the role of heating in the absorption modelling. Verifying the correct absorption mechanism is decisive in providing a better understanding of the complex behaviour of the warm dense state.
\end{abstract}

An important challenge for modern theoretical physics is the description of warm dense matter which is present in dense astrophysical plasmas [1,2], material science [3], and inertial confinement fusion schemes [4]. The complex nature of these energetic states stems from the coexistence of partial degeneracy, strong particle coupling and excited electrons. However, since the bulk properties of matter are ultimately connected to the microscopic structure and dynamics, experiments measuring such properties can act as a gateway to better understanding. The collisional or inverse bremsstrahlung absorption of extreme ultraviolet radiation (XUV) by warm dense matter is one such route to uncovering properties such as the electron-ion collision rate, and is a subject of great interest. Here, we describe novel experimental evidence for the absorption of $25-30 \mathrm{eV}$ photons in solid density warm aluminium $\left(T_{e} \approx 1 \mathrm{eV}\right)$. These results help distinguish between the weak scattering model of Vinko et al. [5] and the corrected classical model of Iglesias [6], which show distinctly different behaviours with temperature. Aluminium is an oft chosen prototypical material used to study the behaviour of matter under warm dense conditions. Also, as the XUV radiation we study here lies above the aluminium plasma frequency $(\approx 15$ $\mathrm{eV}$ photons), yet below the L-edge $(72.6 \mathrm{eV})$, we are assured that free-free absorption is the dominant mechanism for transferring energy to the medium, making it a perfect candidate for testing collisional XUV absorption processes.

The experiment was performed at the Vulcan laser facility (UK) [7]. Figure 1 shows a schematic of the target setup. A sub-micron aluminium sample foil is supported by a thin steel frame and placed at $45^{\circ}$ between two palladium coated $\mathrm{CH}$ foils, $1 \mathrm{~mm}$ from each. Laser pulses of $\approx 2 \times 10^{15} \mathrm{~W} / \mathrm{cm}^{2}$ strike the palladium foils, converting the incident light energy into M-L band x-rays in the $3-3.5 \mathrm{keV}$ region, with an efficiency of approximately 4 $\%[8,9]$. These $\mathrm{keV}$ x-rays are used to volumetrically heat the thin aluminium foil to create a core of solid density warm dense aluminium that can be probed by a short duration pulse of XUV light. The $\mathrm{CH}$ backing suppresses lower energy photons to ensure more volumetric heating of the sample. Four crystal based x-ray spectrometers, an x-ray pinhole camera, and an x-ray streak camera are used to characterise the $\mathrm{keV}$ x-rays from the palladium foils. This technique rapidly deposits energy before substantial hydrodynamic expansion of the aluminium occurs and has previously been studied by the authors [8]. Ray tracing simulations show uniform solid density samples can be created, with temperatures in the warm dense matter regime and a maximum variation of $\approx 10 \%$ in the temperature across the sample face. The aluminium sample will have a uniform surface layer of a few $\mathrm{nm}$ of oxide [10] and typically a few $\mathrm{nm}$ of organic surface contamination [11]. In addition, despite the rapid $\mathrm{x}$-ray heating, the outer edges of the aluminium foil will begin to decompress during heating. Due to these surface factors, the experiment relies upon probing different thicknesses of foil on separate shots and noting that the difference in transmission between any two samples is due solely to the additional thickness of solid density warm aluminium. 


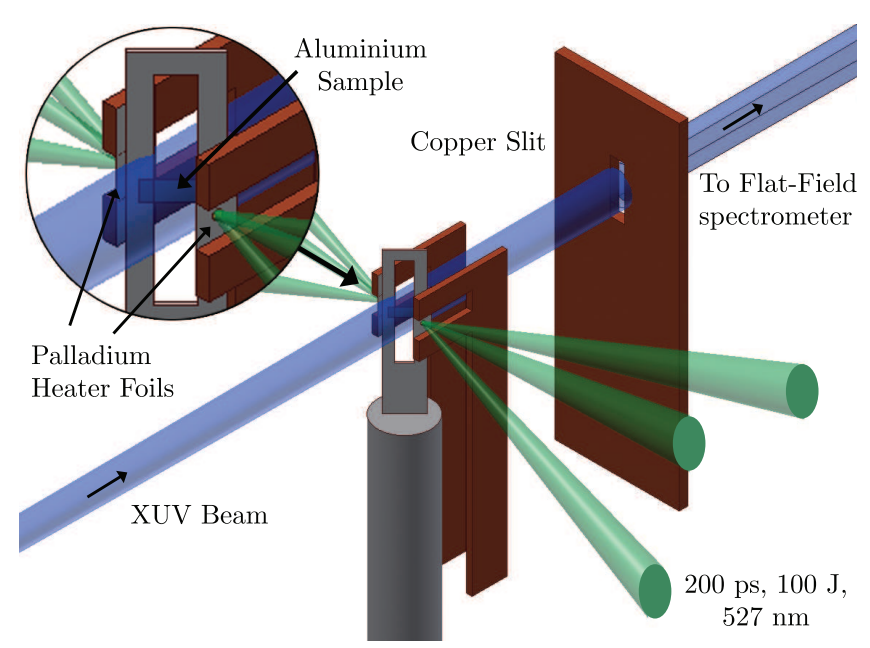

FIG. 1. (Color online) Experiment Setup. Two palladium coated $\mathrm{CH}$ foils surround a thin sub-micron aluminium sample (1mm away). Three $527 \mathrm{~nm}$ laser pulses provide a total of $\approx 2 \times 10^{15} \mathrm{~W} / \mathrm{cm}^{2}$ laser intensity onto each palladium foil (100 J in 200 ps FWHM), which is converted into hard $\mathrm{x}-$ rays that bathe the sample, raising its temperature. An XUV probe propagates through the heated sample and onwards to a spectrometer for analysis.

The XUV probe is generated by a synchronised short pulse laser $(\approx 1 \mathrm{ps}, 527 \mathrm{~nm})$ which is loosely focused through an argon gas jet to create a bright source of high harmonics $[12,13]$, providing discrete spectral lines of radiation in the $40-60 \mathrm{~nm}$ region $(20-30 \mathrm{eV})$. The probe beam is P-polarised with respect to the target angle and propagates through and around the sample, before being spectrally dispersed inside a flat-field grating spectrometer [14] for analysis. A copper shield restricts the probe rays to those that pass through the sample frame. It should be noted that such foil heating and XUV probing studies cannot be easily achieved on existing XUV-FEL experiments, which are excellent for cold matter studies.

Data from an XUV shot through a heated aluminium sample is shown in figure 2 (a). Three harmonic orders can be seen; the 13th, 11th and 9th (from top to bottom), with the 11th order being the brightest by a factor of nearly four. The presence of the sample foil can clearly be seen in the centre of the profile where there is a large drop in signal. Carbon emission lines from the $\mathrm{CH}$ backing on the palladium (in higher diffraction order) as well as low-level continuum emission background are visible, but consistent (less than $1 \%$ noise level with respect to the main signal) and easily identified for removal from the spectra. Unfortunately due to the effects of refraction upon penetrating through the sample foil, the data from the 9th harmonic is unreliable. This is discussed later in the text.

In figure 2 (b) an averaged lineout profile along the spatial axis is given for the 11th harmonic on a shot through an $838 \mathrm{~nm}$ cold aluminium sample. The sam-
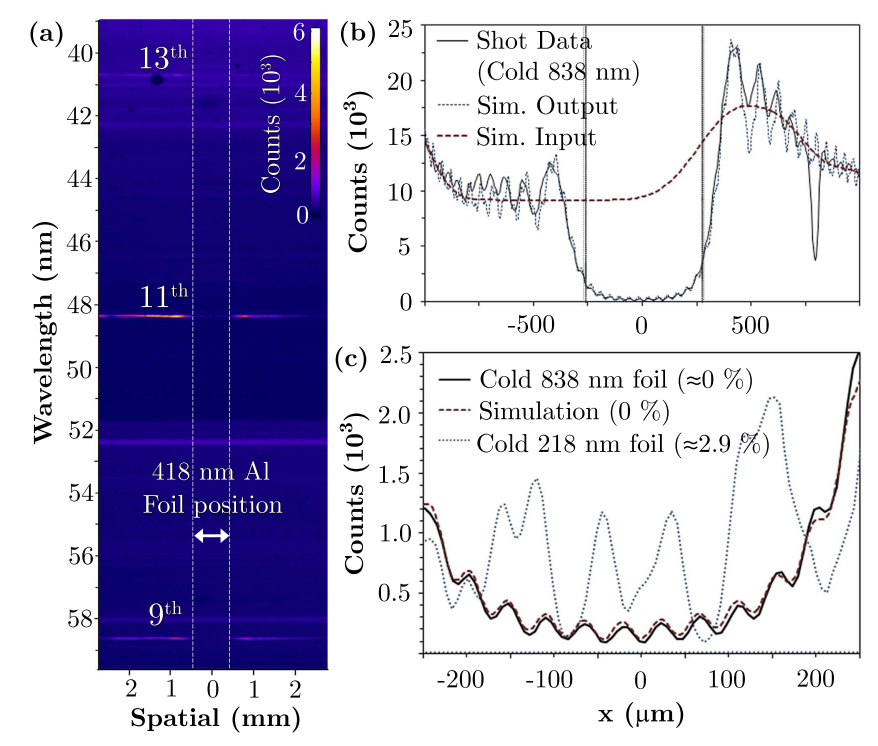

FIG. 2. (Color online) (a) Spectrometer data for an XUV shot through a heated $418 \mathrm{~nm}$ foil, with the position of the sample foil in the spatial axis (horizontal) highlighted. The spectral dispersion in the image goes from top to bottom, with increasing wavelength (or decreasing photon energy). (b) A lineout along the spatial axis of the 11th harmonic, from a shot through a cold $838 \mathrm{~nm}$ aluminium foil, centred at $\mathrm{x}=$ 0 (black solid line). The diffraction simulation input (red dashed line) and output (blue dotted line) are compared to this data. The position of the foil edges are highlighted with the vertical dotted lines. (c) The same shot data of 2 (b) (solid black) compared to the source-broadened output of the diffraction simulation (red dashed line). The shot data for the 11th harmonic on a cold $218 \mathrm{~nm}$ foil is also included (blue dotted line) for comparison.

ple thickness has been accurately measured by RAL Target Fabrication department using a contact probe and is known to $\pm 5 \mathrm{~nm}$. There is evidence of diffraction effects both outside and inside the foil shadow region. Note that the drop in signal around $\mathrm{x}=800 \mu \mathrm{m}$ is an artefact of the CCD. The diffraction features have been simulated with a mathematical model $[15,16]$ that allows for a nonuniform XUV profile to be incident upon the strip of aluminium foil. If the observation plane is sufficiently close to an object or aperture, we are in the near-field region and Fresnel diffraction laws apply [15, 16]. This is true when $R<\frac{a^{2}}{\lambda}$, where $a$ is the obstacle or aperture width, and $R$ is the smaller of the source-to-obstacle or obstacleto-observation distances. For the experiment described here, $\lambda$ is of the order of $10 \mathrm{~s}$ of nanometres (XUV), $R=1$ $\mathrm{m}$ for the obstacle-to-observation plane (the spectrometer), and $a=500 \mu \mathrm{m}$ (the sample foil width); we are within the near-field regime. We are confident that the transmission of this thicker foil is known to be effectively zero $(<0.1 \%)$ at this photon energy, and this provides us with an unambiguous reference for testing our ability to match the measured diffraction pattern with the 
calculated one. All the integrated energy in the shadow region of the profile is due to diffraction from the edges. This model is applied in figure 2 (b) where we see that the interference pattern positions of the real data compare extremely well to the simulation output. Figure 2 (c) shows a close-up of the foil shadow region, where this time the simulation has a Gaussian broadening (FWHM $38 \mu \mathrm{m}$ ) applied to account for source broadening of the XUV probe. We see the fit is excellent (a $99.7 \%$ match across the foil shadow). Data from a $218 \mathrm{~nm}$ cold foil is also shown, highlighting the effect of some finite level of transmission (in this case $\approx 2.9 \%$ ). The higher signal level is clear, with a modified structure due to interference between the transmitted and diffracted signals.

The harmonic generation of the XUV probe is prone to shot-to-shot variations in total energy and spatial distribution, yet our experimental arrangement did not allow for simultaneous measurement of the beam profile before transmission. However, from studying test shots with no sample in place, it is seen that when a large peak structure is apparent in the XUV profile, the shape is typically Gaussian in nature. We find that by fitting Gaussians to the signal outside the shadow of the foil, we are able to predict the total energy under the peak in the foil shadow region to within $\pm 15 \%$. For this, the data from a test harmonic shot is compared to three fits; a target best fit, and two fits deemed to be the worst case scenarios for an over estimation and an under estimation. The worst case estimations have a normalised $\chi^{2}$ test value twice that of the target fit in the fitting regions. It is the largest source of uncertainty in the transmission measurements. By integrating the total signal detected in the foil shadow region, and subtracting the signal calculated through the opaque diffraction simulation over the same limits, a value for the total transmitted signal can be found. The transmission values for the cold data shots are given in table I.

The values in table I include the effects of surface oxides and contaminants mentioned previously. These layers are extremely difficult to characterise or remove, but must be accounted for in any absorption measurement. We make the assumption here that the surface layer of

TABLE I. Measured transmission values. Errors are the resulting change in transmission after estimating the maximum and minimum incident XUV profiles deemed plausible in the foil shadow region, combined with the error margin in the diffraction simulation.

\begin{tabular}{lllll}
\hline \hline Al Sample & Order & Energy $(\mathrm{eV})$ & Trans. (\%) & \pm Error $(\%)$ \\
\hline Cold 218 nm & 11th & 25.88 & 2.91 & 0.43 \\
& 13th & 30.58 & 5.84 & 0.90 \\
Cold $418 \mathrm{~nm}$ & 11th & 25.88 & 0.96 & 0.15 \\
& 13th & 30.58 & 2.42 & 0.36 \\
Heated 218 nm 11th & 25.88 & 2.83 & 0.40 \\
Heated 418 nm 11th & 25.88 & 1.01 & 0.15 \\
\hline \hline
\end{tabular}

each sample foil being probed is identical (all manufactured in the same manner, at the same time, in the same environment), and thus have an identical transmission. Equation 1 is used to calculate the absorption coefficient for each individual harmonic of frequency $\omega$.

$$
\alpha(\omega)=\frac{\ln \left(T_{a}(\omega) / T_{b}(\omega)\right)}{L_{b}-L_{a}}
$$

where $T_{a}$ and $T_{b}$ is the transmission of the foils of length $L_{a}$ and $L_{b}$, respectively. The length values used here are the optical path length travelled by the probe through the sample foils. It accounts for the refraction of the signal as it is incident to the foil at 45 degrees. The index of refraction used for calculating the deviation of the probe is close to unity for the 11th and 13th harmonic orders, and values are taken from Iglesias [6]. Unfortunately for the 9th order, the index of refraction is much lower and the error margin in said value relates to a large fluctuation in the predicted absorption coefficient.

The absorption coefficients of the cold aluminium were found to be $2.47( \pm 0.69) \times 10^{6} \mathrm{~m}^{-1}$ and $2.44( \pm 0.68) \times$ $10^{6} \mathrm{~m}^{-1}$ for the 11 th and 13th harmonics, respectively. These results are compared (see figure 4 (a) later) to the theoretical models of Vinko et al. [5] and Iglesias [6], and the pre-existing experimental data sets of Keenan et al. [17], Gullikson et al. [18], and Henke et al. [19]. Although the error margins are large, there is strong agreement with the values of Gullikson et al., but yet both theoretical models seem to predict much higher absorptions than those measured. It is true that the models discussed are not fully designed for cold material predictions. We focus our examination on the role of heating and the presence of a relative absorption change with raising sample temperature.

For heated foils, in addition to oxide and contaminant layers we should expect that there will be an expanded sub-solid density plasma on either face of the heated foils. Hydrodynamic simulations were used to estimate the sample foil conditions upon heating with the well characterised palladium x-ray drive. For more information see Kettle et al. [8]. The heating of the foils using this technique predominantly lies in a well understood photon range $(3 \mathrm{keV})$, so the simulations should be reliable in estimating the expansion. For both heater foils, the x-ray yields observed by the crystal based spectrometers on that shot, in combination with a blackbody model, were used to generate the photon flux source for HYADES modelling (a 1-D Lagrangian code) [20]. The density and electron temperature of the two shots being analysed are shown in figure 3 . One shot is a $418 \mathrm{~nm}$ foil, probed after $104 \pm 5 \mathrm{ps}$ and the other is a $218 \mathrm{~nm}$ foil, probed after $108 \pm 5$ ps. The spatial co-ordinates of the $218 \mathrm{~nm}$ foil have been split along its centre to move both edges to overlap those of the $418 \mathrm{~nm}$ target. The thickness of each foil is increased by $1 / \cos 45^{\circ}$ to allow for the fact that the XUV probe penetrates the targets at 


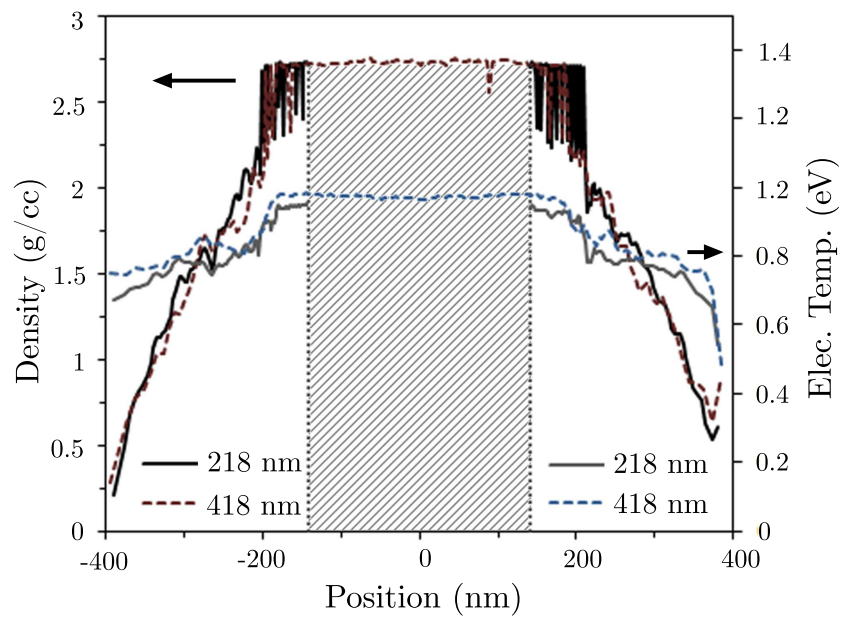

FIG. 3. (Color online) Target sample conditions. A comparison of the $418 \mathrm{~nm}$ (dashed lines) and $218 \mathrm{~nm}$ (solid lines) target conditions, where the $218 \mathrm{~nm}$ foil has been split along its centre and shifted to match the edge position of the 418 $\mathrm{nm}$ foil. The additional material of the $418 \mathrm{~nm}$ target is highlighted in crosshatch. The density and electron temperature are given by the left-hand and right-hand axis, respectively.

$45^{\circ}$, with a corresponding increase in the presented foil thickness. The additional $283 \mathrm{~nm}$ of material (initial difference in thickness at $45^{\circ}$ ) in the $418 \mathrm{~nm}$ case has been highlighted in both plots. The heating in this region is extremely uniform, around $1 \mathrm{eV}$, and the foil remains at solid density at the time of probing and so is suitable for comparison to the models of Vinko et al. and Iglesias. We can see that the expansion at the edges of the target is similar for both, and note that any difference in target transmission should be due to only the additional central material of the $418 \mathrm{~nm}$ target. Although the classical absorption coefficient is not expected to be accurate in a great deal of the sub-solid density plasma, we can use it to compare relatively the two cases and see that the predicted absorption is within $8 \%$ of each other [24]. This figure is used to estimate the potential error bar in assuming the transmission of the expanded plasmas is equivalent for the two foil thicknesses.

The transmission values for both heated shots were found using the same method as that which was used for the cold foils. Table I details the measurements. Iglesias shows that the index of refraction value used to calculate the optical path length for the coefficient calculation should not change upon heating (below $10 \mathrm{eV}$ ). For the 11th harmonic the absorption coefficient was found to be $2.30( \pm 0.64) \times 10^{6} \mathrm{~m}^{-1}$; similar to the cold foil value. The results indicate that upon heating the XUV absorption of the aluminium remains virtually unchanged. Unfortunately, a measurement of the 13th harmonic was not possible for either of the heated foils as the signal was below the detection limit. In figure 4 (b) we compare the measured warm dense absorption coefficient, normalised
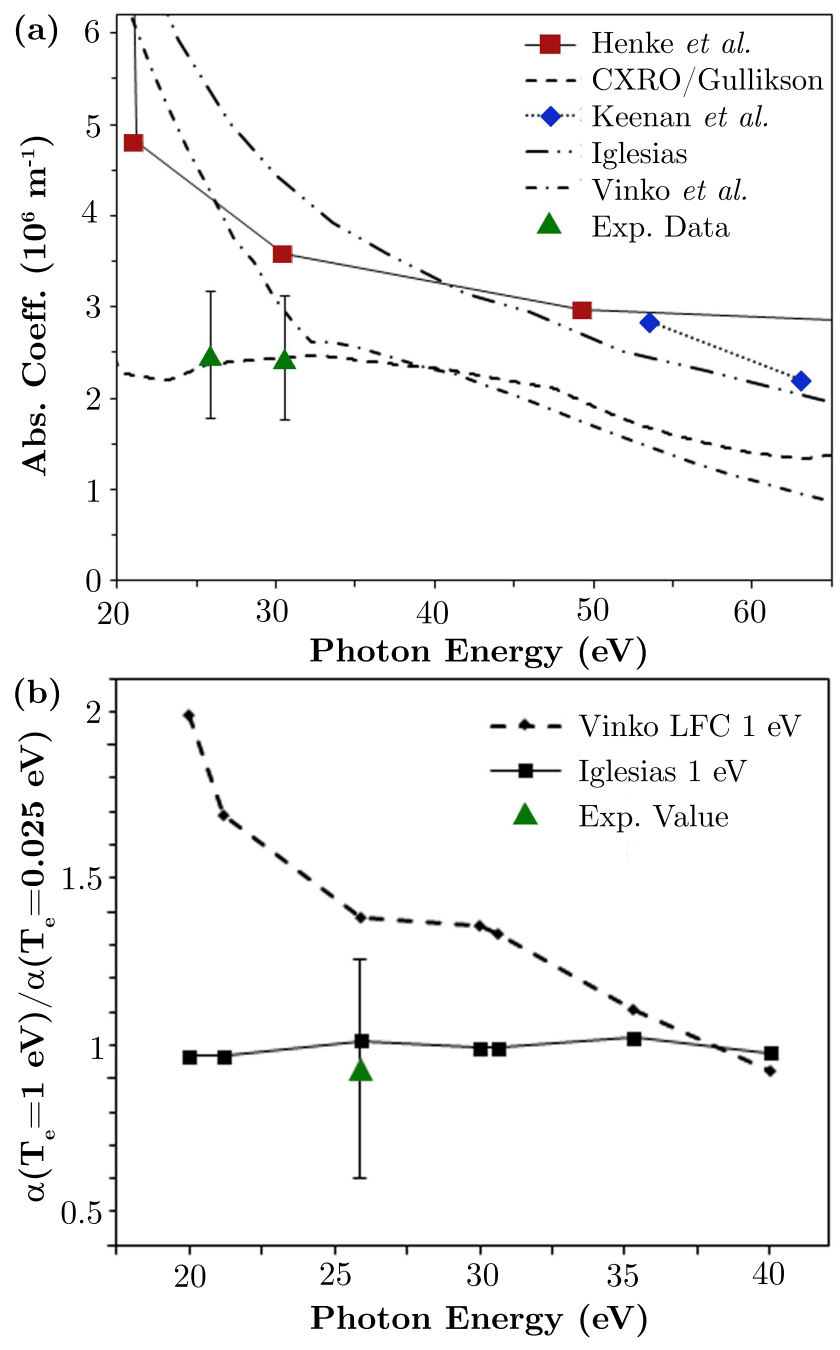

FIG. 4. (Color online) (a) Measured absorption coefficients of cold aluminium (green triangles), compared to existing model predictions and experimental data. (b) Warm dense absorption coefficients (normalised to the cold values) compared to the normalised coefficients for both the Vinko et al. and Iglesias models under similar conditions (solid density, $T_{e} \approx$ $1 \mathrm{eV})$. The errors bars were calculated using the standard combination in quadrature method $\left(\Delta E=\sqrt{\Delta A^{2}+\Delta B^{2}}\right.$ where $\Delta E$ is the resulting error and $\Delta A$ and $\Delta B$ are the errors in the two transmission measurements respectively).

to the measured cold value, to that of the Iglesias and Vinko et al. models (both of which are normalised to their cold absorption coefficient values). We note that by comparing the normalised absorption of cold and heated material, as the optical path length should not change, we should not be worried by any error in assuming the index of refraction value. The result indicates that the Iglesias model more accurately predicts the absorption upon heating.

In summary, upon heating, the absorption coefficient of $T_{e} \approx 1 \mathrm{eV}$ solid density aluminium remains very similar to its absorption under cold ambient conditions, for 
$25.9 \mathrm{eV}$ photons (the 11th harmonic order). This is a significant result as the Vinko et al. model predicts an increase in absorption of 40-70 \% for these photon energies, whereas the Iglesias model predicts no change. Iglesias elaborates further on the possible source of the difference from Vinko et al. $[22,23]$. Using two different calculations for the electron-ion interaction, one based on the usual all-order interaction formula corrected for degeneracy and many-body screening, and another with a Born (or weak scattering) approximation, Iglesias suggests that the weak scattering approximation used by Vinko et al. may partly explain the discrepancy in the rate of absorption change with temperature. The increase in absorption with higher temperature (above $T_{e} \approx 4-5 \mathrm{eV}$ ) in the corrected classical model is due to an increase in the many-body screening length and a reduction in the electron degeneracy. We have also observed that the measured cold absorption coefficient is lower than both models discussed. This may highlight a deficiency in the models that must be addressed. Iglesias briefly suggests that including the dynamic response of the bound electron, instead of a frozen-core potential, may improve agreement. Further experimental measurements in the XUV photon region would be extremely useful in clarifying this discussion.

In conclusion, the novel experimental method described has proved extremely useful in providing evidence to explore the two inverse bremsstrahlung models discussed. Further work, with a refined experimental method allowing for simultaneous input and transmitted profile measurement, though challenging, would provide a greater insight into the true behaviour of the collisional absorption of not only aluminium, but other low-Z materials.

This work was supported by the Engineering and Physical Sciences Research Council (grants EP/I031464 and EP/I029206/1), European program COST MP1203, and the Science and Technology Facilities Council (STFC), UK. We acknowledge the support and contribution of the Target Fabrication Laboratory, Vulcan laser staff and the Engineering workshop at CLF, RAL.

\footnotetext{
* Corresponding author: bkettle01@qub.ac.uk
}

[1] M. Koenig et al., Nucl. Fusion 44, S208-S214 (2004).

[2] N. Nettelmann, R. Redmer and D. Blaschke, Physics of Particles and Nuclei 39, 1122-1127 (2008).

[3] R. Ernstorfer et al., Science 323, 10331037 (2009).

[4] S. Glenzer et al., Science 327, 12281231 (2010).

[5] S. Vinko et al., High Energy Density Physics 5, 124-131 (2009).

[6] C. A. Iglesias, High Energy Density Physics 6, 311-317 (2010).

[7] I. N. Ross et al., IEEE J. Quantum Electron. 17, 16531661 (1981).

[8] B. Kettle et al., J. Phys. B 48, 224002 (2015).

[9] D. W. Phillion and C. J. Hailey, Phys. Rev. A 34, 48864896 (1986).

[10] D. Hemmers, M Benzid and G. Pretzler, Appl. Phys. B 108, 167175 (2012).

[11] R. Robinson, BSc thesis, Brigham Young University (2003), available at http://volta.byu.edu/pubs/RossCleaning.pdf

[12] T. Ditmire, J. K. Crane, H. Nguyen, L. B. DaSilva and M. D. Perry, Phys. Rev. A 51, R902-R905 (1995).

[13] C.-G. Wahlstrm et al., Phys. Rev. A 48, 4709-4720 (1993).

[14] T. Kita, T. Harada, N. Nakano and H. Kuroda, Appl. Opt. 22, 512-513 (1983).

[15] E. Hecht, Optics (Addison-Wesley, Boston, 4th edition, 2002).

[16] M. Born and E. Wolf, Principles Of Optics (Pergamon Press, Oxford, 4th edition, 1970).

[17] R. Keenan, C. L. S. Lewis, J. S. Wark and E. Wolfrum, J. Phys. B 35, L447-L451 (2002).

[18] E. M. Gullikson, P. Denham, S. Mrowka and J. H. Underwood, Phys. Rev. B 49, 16283-16288 (1994).

[19] B. L. Henke, E. M. Gullikson and J. C. Davis, At. Data and Nucl. Data Tables 54, 181-342 (1993).

[20] J. T. Larsen and S. M. Lane, J. Quant. Spectrosc. Radiat. Transfer 51, 179 (1994).

[21] Center for X-ray Optics, X-ray Database; http://henke.lbl.gov/optical_constants/

[22] C. A. Iglesias, High Energy Density Physics 7, 38-39 (2011).

[23] S. M. Vinko, G. Gregori and J. S. Wark, High Energy Density Physics 7, 40-42 (2011).

[24] L. B. Da Silva et al., Phys. Rev. Lett. 74, 3991-3994 (1995). 